\title{
Linear response theory in the continuum for deformed nuclei: Green's function vs time-dependent Hartree-Fock with the absorbing boundary condition
}

\author{
Takashi Nakatsukasa and Kazuhiro Yabana \\ Center for Computational Sciences and Institute of Physics, University of Tsukuba, Tsukuba 305-8571, Japan
}

(Received 7 September 2004; published 1 February 2005)

\begin{abstract}
The continuum random-phase approximation is extended to the one applicable to deformed nuclei. We propose two different approaches. One is based on the use of the three-dimensional (3D) Green's function, and the other is the small-amplitude TDHF with the absorbing boundary condition. Both methods are based on the 3D Cartesian grid representation and applicable to systems with no symmetry on nuclear shape. The accuracy and identity of these two methods are examined with the BKN interaction. Using the full Skyrme energy functional in the small-amplitude TDHF approach, we study the isovector giant dipole states in the continuum for ${ }^{16} \mathrm{O}$ and for even-even Be isotopes.
\end{abstract}

DOI: 10.1103/PhysRevC.71.024301

PACS number(s): 21.60.Jz, 21.10.Pc, 27.20.+n

\section{INTRODUCTION}

Mean-field theories with effective interactions [1-4] have been extensively used for systematic description of nuclear ground-state properties from light to heavy nuclei, including infinite nuclear matter. Nuclear mass, radius, density distribution, and deformation are the primary target of the static effective mean-field theory [5]. The concept of the nuclear mean-field theory is rather different from the Hartree-Fock theory in electronic systems but is more close to the density functional theory. Especially, the Hartree-Fock (HF) with the zero-range Skyrme interaction results in an energy functional of local densities. A similar form of functional was obtained from the density-matrix expansion of energy functionals calculated with the microscopic nucleon-nucleon forces [6,7].

Although the static mean-field calculations well reproduce the bulk nuclear properties throughout the nuclear chart, it is necessary to go beyond the mean field to describe excited states and correlations associated with many kinds of collective motions. The generator coordinate method (GCM) $[8,9]$ is one of the standard methods to take account of the configuration mixing. The GCM based on the mean-field theory provides a unified description of single-particle and collective nuclear dynamics. In practice, collective variables, $q$, are chosen from physical intuition and are restricted to one dimension in most cases. For instance, to describe quadrupole excitations, the most common choice is the mass quadrupole moment, $q=$ $A\left\langle\Phi(q)\left|r^{2} Y_{20}\right| \Phi(q)\right\rangle$, where the single-Slater states $|\Phi(q)\rangle$ are determined by the constrained Hartree-Fock(-Bogoliubov) calculation. This is a drawback of the GCM that one has to prepare, a priori, a set of states $\{|\Phi(q)\rangle\}$.

The time-dependent Hartree-Fock (TDHF) theory is a complementary method to the GCM. The system determines its collective path for itself and the TDHF takes care of both collective and single-particle excitations. The TDHF is also known to produce the proper inertial parameters [10] because it is a dynamical theory to incorporate time-odd components in the wave function. A drawback is its semiclassical nature. Namely, to calculate quantal quantities, such as eigenenergy and transition probability, one has to requantize obtained TDHF dynamics. Although it is a difficult task to requantize the TDHF orbitals in general [11], the perturbative regime can be easily handled. The linear approximation leads to the random-phase approximation (RPA) for the effective densitydependent forces, which is analogous to the time-dependent local-density approximation in electronic systems $[12,13]$. Another advantage of TDHF is its ability to describe spreading width of collective motion induced by the interaction between particles and time-dependent mean-field potential (one-body dissipation). The escape width can be also described by the TDHF but requires proper treatment of the continuum. In this article, we propose a feasible method to treat the continuum in the real-space TDHF calculation. That is the absorbing boundary condition $(\mathrm{ABC})$ approach. We have already studied photoabsorption in molecules [14] and nuclear breakup reaction $[15,16]$ with the similar technique. Our earlier attempts for nuclear response calculation have been reported in Refs. [17-20].

The Green's function method in the linear response proposed by Shlomo and Bertsch [21] is a common way to treat the continuum boundary condition. It is usually called "continuum RPA" in nuclear physics. The same idea was proposed later in the time-dependent density functional theory (TDDFT) for calculations of photoresponse in rare-gas atoms [22]. The method has been widely applied to spherical (magic or semimagic) nuclei [23-32]; however, its application to deformed systems has not been done so far, because the explicit construction of Green's function is extremely difficult for deformed potential. We have recently proposed an iterative method to construct response functions for deformed systems with the proper boundary condition in the three-dimensional (3D) coordinate space and studied molecular photoabsorption using the TDDFT $[14,33]$. There, the dynamical screening effect in the continuum for a multicenter problem was a key issue for understanding the photoabsorption cross section at photon energies higher than the ionization potential. The same method is applicable to nuclear mean-field models that do not contain nonlocal densities. In this respect, applications to the Skyrme energy functional is parallel to the TDDFT. In this article, we extend a method of the continuum RPA to the one in the $3 \mathrm{D}$ coordinate space and apply it to deformed nuclei. 
We call this "3D continuum RPA" in this article. This provides the exact treatment of the nucleonic continuum for deformed nuclei [34]. The results can be used to check validity of the $\mathrm{ABC}$ approach.

Another issue addressed in this article is the self-consistent treatment in the continuum response calculation. The nuclear energy functional is far more complicated than that of electronic TDDFT. The Skyrme functional is one of the simplest, because its nonlocal part is expressed by derivatives of local densities. Even so, the continuum RPA calculations so far neglect the spin-orbit and Coulomb residual particle-hole interactions, which violates the self-consistency with the HF field $[29,35]$. In addition, a time-reversal-odd (time-odd) part of densities, such as spin densities, are often omitted. Because the spin-orbit term in the time-even mean field is related to spin-current terms in the time-odd mean field by the local gauge (Galilean) invariance [36,37], the neglect of spin density violates this symmetry. As far as we know, at present, there is no fully self-consistent Skyrme-HF-based continuum RPA calculations, even for spherical nuclei. We perform the smallamplitude TDHF calculation with the $\mathrm{ABC}(\mathrm{TDHF}+\mathrm{ABC})$ in fully self-consistent manner for the giant dipole resonance in ${ }^{16} \mathrm{O}$ and examine effect of residual interactions that have been neglected so far. In the time-dependent relativistic meanfield approach without the continuum, the small-amplitude real-time calculation has been attempted for spherical nuclei $[38,39]$. However, only a very short time period $(3 \sim$ $4 \hbar \mathrm{MeV}^{-1}$ ) was achieved, which prevents them from carrying out a quantitative analysis. See also recent articles [40-42] and references therein for the present status of the self-consistent $\mathrm{HF}(\mathrm{B})+(\mathrm{Q}) \mathrm{RPA}$ calculations for spherical nuclei. It should be noted that, without the continuum boundary condition, there exist a few works of fully self-consistent RPA for deformed nuclei calculated in the 3D coordinate space with the full Skyrme interaction $[43,44]$.

In recent developments of radioactive-ion-beam facilities, the Coulomb excitation and the inelastic scattering are becoming standard methods to investigate excited states in unstable nuclei. For weakly bound systems, the treatment of the continuum should be extremely important. Moreover, we know most of open-shell nuclei are deformed. Collective modes of excitation in the particle continuum in deformed nuclei become the main interest in those studies. This has not been examined in a self-consistent manner so far. The present article provides us with general methods of linear response in the continuum for systems whose energy functional is given by local one-body densities.

The article is organized as follows: Section II presents a method of extending the continuum RPA to deformed nuclei. In Sec. III, we present a real-time TDHF method using the absorbing boundary condition. Some illustrative examples show effect of the continuum and comparison between these two methods in Sec. IV. In Sec. V, we present numerical results of the small-amplitude $\mathrm{TDHF}+\mathrm{ABC}$ calculation in real time using the Skyrme energy functional for giant dipole resonances. Effects of time-odd densities, and $E 1$ strengths in ${ }^{16} \mathrm{O}$ and those in neutron-rich $\mathrm{Be}$ isotopes are discussed. The conclusion is summarized in Sec. VI.

\section{3D CONTINUUM RPA}

For spherical systems, the continuum RPA is formulated in terms of the radial Green's function using a multipole expansion [21]. Hereafter, we refer to this as "1D continuum RPA." In Ref. [14], we have presented a method to construct a Green's function in the 3D grid representation for a system without any spatial symmetry. In this section, we recapitulate the method of constructing the response function. Spin and isospin indices are suppressed for simplicity and $\hbar=1$ is used.

The HF Hamiltonian, $h[\rho]$, is a functional of one-body density matrix [45]. In case of zero-range effective interactions, it is a functional of local one-body density $\rho(\mathbf{r})$. The stationary condition is as follows:

$$
[h, \rho]=0,
$$

which defines the HF ground state density $\rho=\rho_{0}$. Then, the TDHF equation with an external perturbation,

$$
i \frac{d}{d t} \rho(t)=\left[h[\rho]+V_{\mathrm{ext}}(t), \rho(t)\right],
$$

is linearized with respect to the density fluctuation as follows:

$$
\rho(\mathbf{r}, t)=\rho_{0}(\mathbf{r})+\delta \rho(\mathbf{r}, t) .
$$

This leads to the well-known RPA equation. The transition density, $\delta \rho(\mathbf{r} ; \omega)$, which is the Fourier transform of $\delta \rho(\mathbf{r}, t)$, can be expressed as follows $[22,35]$ :

$$
\begin{aligned}
\delta \rho(\mathbf{r} ; \omega)= & \iint d \mathbf{r} \Pi\left(\mathbf{r}, \mathbf{r}^{\prime} ; \omega\right) V_{\mathrm{ext}}\left(\mathbf{r}^{\prime} ; \omega\right) \\
= & \int d \mathbf{r}^{\prime} \Pi_{0}\left(\mathbf{r}, \mathbf{r}^{\prime} ; \omega\right) \\
& \times\left(V_{\mathrm{ext}}\left(\mathbf{r}^{\prime} ; \omega\right)+\int d \mathbf{r}^{\prime \prime} v\left(\mathbf{r}^{\prime}, \mathbf{r}^{\prime \prime}\right) \delta \rho\left(\mathbf{r}^{\prime \prime} ; \omega\right)\right),
\end{aligned}
$$

where $\Pi$ and $\Pi_{0}$ are the RPA and the independent-particle response function, respectively. The $v\left(\mathbf{r}, \mathbf{r}^{\prime}\right)$ is a residual interaction which is defined by the following:

$$
v\left(\mathbf{r}, \mathbf{r}^{\prime}\right) \equiv \frac{\delta^{2} E[\rho]}{\delta \rho(\mathbf{r}) \delta \rho\left(\mathbf{r}^{\prime}\right)} .
$$

Here, we assume that the total energy functional, $E[\rho]$, is a function of local density $\rho(\mathbf{r})$ only. The HF mean field is also local in the coordinate space. Assuming that a one-particle moment $F(\mathbf{r})$ depends only on the spatial coordinates, the transition strength is obtained from the transition density as follows:

$$
\begin{aligned}
\frac{d B(\omega ; F)}{d \omega} & \equiv \sum_{n}|\langle n|F| 0\rangle|^{2} \delta\left(\omega-E_{n}\right) \\
& =-\frac{1}{\pi} \operatorname{Im} \iint d \mathbf{r} d \mathbf{r}^{\prime} F(\mathbf{r}) \Pi\left(\mathbf{r}, \mathbf{r}^{\prime} ; \omega\right) F\left(\mathbf{r}^{\prime}\right) \\
& =-\left.\frac{1}{\pi} \operatorname{Im} \int d \mathbf{r} F(\mathbf{r}) \delta \rho(\mathbf{r} ; \omega)\right|_{V_{\text {ext }}=F} .
\end{aligned}
$$

In case of deformed nuclei, $|0\rangle$ and $|n\rangle$ are not eigenstates of total angular momentum operator. Thus, $d B(\omega ; F) / d \omega$ should be regarded as the intrinsic transition strength. In Sec. VB, we assume the strong coupling scheme [46] to transform 
calculated intrinsic strength to the quantity in the laboratory frame. The response function is written as follows:

$$
\begin{aligned}
& \Pi\left(\mathbf{r}, \mathbf{r}^{\prime} ; \omega\right)=\Pi_{0}\left(\mathbf{r}, \mathbf{r}^{\prime} ; \omega\right) \\
& \quad+\iint d \mathbf{r}^{\prime \prime} d \mathbf{r}^{\prime \prime \prime} \Pi_{0}\left(\mathbf{r}, \mathbf{r}^{\prime \prime} ; \omega\right) v\left(\mathbf{r}^{\prime \prime}, \mathbf{r}^{\prime \prime \prime}\right) \Pi\left(\mathbf{r}^{\prime \prime \prime}, \mathbf{r}^{\prime} ; \omega\right), \\
& \Pi_{0}\left(\mathbf{r}, \mathbf{r}^{\prime} ; \omega\right)=\sum_{i=1}^{A}\left\{\phi_{i}(\mathbf{r}) G^{(-)}\left(\mathbf{r}, \mathbf{r}^{\prime} ; \epsilon_{i}-\omega\right) \phi_{i}^{*}\left(\mathbf{r}^{\prime}\right)\right. \\
& \left.\quad+\phi_{i}^{*}(\mathbf{r}) G^{(+)}\left(\mathbf{r}, \mathbf{r}^{\prime} ; \epsilon_{i}+\omega\right) \phi_{i}\left(\mathbf{r}^{\prime}\right)\right\} .
\end{aligned}
$$

The single-particle Green's function in Eq. (11) is defined by the following:

$$
G^{( \pm)}\left(\mathbf{r}, \mathbf{r}^{\prime} ; E\right)=\left\langle\mathbf{r}\left|\left(E-h\left[\rho_{0}\right] \pm i \eta\right)^{-1}\right| \mathbf{r}^{\prime}\right\rangle .
$$

Here, the superscript $+(-)$ indicates the outgoing (incoming) boundary condition. In case that $h\left[\rho_{0}\right]$ is rotationally invariant, the Green's function of Eq. (12) can be constructed by using the partial-wave expansion as follows:

$$
G^{( \pm)}\left(\mathbf{r}, \mathbf{r}^{\prime} ; E\right)=2 m \sum_{l m} Y_{l m}(\hat{\mathbf{r}}) \frac{u_{l}\left(r_{<}\right) w_{l}^{( \pm)}\left(r_{>}\right)}{W\left[u_{l}, w_{l}^{( \pm)}\right] r r^{\prime}} Y_{l m}^{*}\left(\hat{\mathbf{r}}^{\prime}\right) .
$$

Here, $W$ is the Wronskian and $u_{l}$ and $w_{l}$ are solutions of the radial Schrödinger equation for $h\left[\rho_{0}\right]=-\nabla^{2} / 2 m+V(r)$ :

$$
\left(E+\frac{1}{2 m} \frac{d^{2}}{d r^{2}}-\frac{l(l+1)}{2 m r^{2}}-V(r)\right) R_{l}(r)=0 .
$$

$u_{l}$ is regular at origin and $w_{l}^{( \pm)}$has an outgoing/incoming asymptotic form. In the 1D continuum RPA [21], Eq. (10) is also expanded in partial waves. Then, the 1D RPA response function (in the radial coordinate) is explicitly constructed by using Eqs. (10), (11), and (13).

There are some difficulties to extend the theory to nonspherical systems. The first one is a purely numerical difficulty. Because the number of spatial grid points in the 3D space is much larger than that of the radial grid points, it is hard to explicitly construct the response function, $\Pi\left(\mathbf{r}, \mathbf{r}^{\prime} ; \omega\right)$, and to perform spatial multifold integration. We also need to calculate an inverse matrix to solve Eq. (10). The second difficulty lies in the complexity of boundary condition. Equations (13) and (14) cannot be used for cases of a deformed HF potential.

We solve the first numerical problem by using an iterative procedure for implicit calculation of the response and Green's function. For instance, to calculate the transition density, we recast Eq. (5) into an integral equation for $\delta \rho$ as follows:

$$
\begin{gathered}
\int d \mathbf{r}^{\prime \prime}\left\{\delta\left(\mathbf{r}-\mathbf{r}^{\prime \prime}\right)-\int d \mathbf{r}^{\prime} \Pi_{0}\left(\mathbf{r}, \mathbf{r}^{\prime} ; \omega\right) v\left(\mathbf{r}^{\prime}, \mathbf{r}^{\prime \prime}\right)\right\} \delta \rho\left(\mathbf{r}^{\prime \prime} ; \omega\right) \\
=\int d^{3} r^{\prime} \Pi_{0}\left(\mathbf{r}, \mathbf{r}^{\prime} ; \omega\right) V_{\mathrm{ext}}\left(\mathbf{r}^{\prime} ; \omega\right)
\end{gathered}
$$

This is equivalent to a linear algebraic equation in the 3D grid space and we use the iterative method to solve it. For the linear algebraic problem, $A|x\rangle=|b\rangle$, the iterative methods require neither a full knowledge of the matrix $A$ nor an inverse matrix $A^{-1}$ but do require only results of operating $A$ on a certain vector $|y\rangle$. This means that we do not need to calculate an explicit form of $\Pi_{0}\left(\mathbf{r}, \mathbf{r}^{\prime} ; \omega\right)$. All we need to calculate is the action of $\Pi_{0}$; that is, $\Pi_{0} \cdot v \cdot \delta \rho$ and $\Pi_{0} \cdot V_{\text {ext }}$, where the dot indicates the integral in Eq. (15). This is an advantage of the iterative method over the direct method. In addition, the iterative method is known to be very efficient for a large sparse matrix. Then, the next task is to calculate action of $\Pi_{0}$. According to Eq. (11), we have to operate $G^{ \pm}(E)$ on certain states $|y\rangle$. Now the problem is coupled to the second difficulty, that is, the continuum boundary condition for deformed systems.

We start to divide the HF potential into a long-range spherical part and a short-range deformed one, $h\left[\rho_{0}\right]=$ $-\nabla^{2} / 2 m+V_{0}(r)+\tilde{V}(\mathbf{r})$. In the present work, $V_{0}(r)$ is taken as the Coulomb potential of a sphere of radius $1.2 A^{1 / 3} \mathrm{fm}$ with a uniform change $Z e$. The single-particle Green's function for $h_{0}=-\nabla^{2} / 2 m+V_{0}(\boldsymbol{r})$ is constructed in the same way as Eq. (13), which is denoted by $G_{0}^{( \pm)}(E)$ below. We have an identity for $G$,

$$
\begin{aligned}
& G^{( \pm)}\left(\mathbf{r}, \mathbf{r}^{\prime} ; E\right)=G_{0}^{( \pm)}\left(\mathbf{r}, \mathbf{r}^{\prime} ; E\right) \\
& \quad+\int d^{3} r^{\prime \prime} G_{0}^{( \pm)}\left(\mathbf{r}, \mathbf{r}^{\prime \prime} ; E\right) \tilde{V}\left(\mathbf{r}^{\prime \prime}\right) G^{( \pm)}\left(\mathbf{r}^{\prime \prime}, \mathbf{r}^{\prime} ; E\right)
\end{aligned}
$$

The boundary condition of $G_{0}^{( \pm)}$determines an asymptotic behavior of $G^{( \pm)}$. The action of $G^{( \pm)},\left|x^{( \pm)}\right\rangle=G^{( \pm)}|y\rangle$ for a given state $|y\rangle$, is obtained by solving a linear algebraic equation as follows:

$$
\left\{1-G_{0}^{( \pm)} \tilde{V}\right\}\left|x^{( \pm)}\right\rangle=G_{0}^{( \pm)}|y\rangle .
$$

Here, we use, again, the iterative method to solve this equation.

In summary, to obtain the transition density, we solve Eq. (15). To do this, we need to calculate the operation of $\Pi_{0}$, which then requires us to solve Eq. (17) with a proper boundary condition. The procedure results in multiple-nested linear algebraic equations that are solved with iterative methods, such as the conjugate gradient method. The detailed algorithm is given in Ref. [14].

\section{REAL-TIME TDHF+ABC}

\section{A. Absorbing boundary condition (ABC)}

The TDHF equation can be efficiently solved in the 3D lattice space in real time $[11,47,48]$. The same technique has been applied to TDDFT of finite $[49,50]$ and infinite electronic systems [51]. In the real-time calculation, we propagate singleparticle wave functions $\left\{\phi_{i}\right\}_{i=1, \ldots, A}$ using the same technique as that in Ref. [47].

$$
\phi_{i}(\mathbf{r}, t+\Delta t)=\exp (-i \Delta t \cdot h[\rho(t+\Delta t / 2)]) \phi_{i}(\mathbf{r}, t),
$$

where the exponential operator is expanded in a power series to $(\Delta t)^{4}$. The time step in following applications is taken as $\Delta t=0.001 \mathrm{MeV}^{-1}$. There are many good reasons for solving the problem in real-time representation. First, the computation algorithm becomes very simple. To make the time evolution, only the operation of the HF Hamiltonian on a certain single-particle state, $h[\rho]|\psi\rangle$, is needed to be calculated, though the self-consistency between $\rho(t)$ and $h[\rho]$ 
brings slight complication. Second, a single calculation of the time evolution provides information for a wide range of energy. Thus, for the strength function in a wide energy region, the real-time calculation is often more efficient than that in the energy domain. Last but not least, the TDHF wave packet in real space in real time gives an intuitive understanding of dynamics. In pioneering works on heavy-ion collisions, the TDHF dynamics nicely demonstrated time evolution of nuclear inelastic scattering [11,47,52].

The TDHF time evolution is relatively easy in the present computer power. The problem is how to impose the continuum boundary condition. The exact treatment of the continuum such as the Green's function method is very difficult (even impossible) in this case, because the energy of escaping particles cannot be determined uniquely. Thus, we attempt an approximate treatment. The usual approach to TDHF in real space is to assume the wave function to be zero at some distance $R$ from the origin, which we call "Box boundary condition" (BBC) hereafter. Then, the time evolution must be completed before a significant portion of the wave reaches the boundary. Seeking higher accuracy, we must employ a larger $R$ value, increasing the computation task. Instead, in this article, we employ the "absorbing boundary condition" (ABC), which introduces a complex absorbing potential outside of the system. The method was first tested by Hamamoto and Mottelson for a schematic one-dimensional model of TDHF calculation [53]. Since then, however, its capability has not been fully examined in nuclear theory. Conversely, in other fields of quantum physics, especially in atomic and molecular collision theories, the method has become one of standard methods for calculations of reactive scattering problems (see a recent review article [54] and references therein). We have demonstrated that the $\mathrm{ABC}$ is able to produce results identical to that of the exact continuum with the Green's function for TDDFT study of photoabsorption in molecules [14]. In nuclear three-body reaction models [15], the method was also tested in detail for deuteron breakup reaction and provides an alternative method to the continuum-discretized-coupled channels (CDCC). A similar approach has been tested to calculate nuclear resonance states in a simple model [55].

The success of the ABC approach is based on its simplicity. Actually, it requires only a minor modification of the real-time TDHF code, simply adding a complex potential, $-i \tilde{\eta}(\mathbf{r})$. We replace the HF Hamiltonian in Eq. (18) by the following:

$$
h[\rho] \longrightarrow h[\rho]-i \tilde{\eta}(\mathbf{r}) .
$$

This prescription is equivalent to the use of Green's function of Eq. (12) in which the infinitesimal imaginary part $\eta$ is replaced by a finite and coordinate-dependent $\tilde{\eta}(\mathbf{r})$. The absorbing potential must be zero in a region where the ground-state density has a finite value, and it is finite outside of the system. Of course, the addition of the complex potential violates the unitarity of time evolution. Thus, the norm of each single-particle state decreases with time, which represents a physical process, the emission of particles.

We adopt the same form of absorptive potential as previous works $[14,15]$. This is a linear dependence on the coordinate $[56,57]$ as follows:

$$
\tilde{\eta}(r)= \begin{cases}0 & \text { for } 0<r<R, \\ i \eta_{0} \frac{r-R}{\Delta r} & \text { for } R<r<R+\Delta r .\end{cases}
$$

The size of the inner model space $(r<R)$ is chosen so that the HF ground state converges within this space. The outer space of width $\Delta r(R<r<R+\Delta r)$ is the absorbing region that should be large enough to prevent reflection of emitted outgoing waves. The condition of a good absorber for a particle with mass $m$ and energy $E$ is given by the following:

$$
7 \frac{E^{1 / 2}}{\Delta r \sqrt{8 m}}<\left|\eta_{0}\right|<\frac{1}{10} \Delta r \sqrt{8 m} E^{3 / 2} .
$$

Here, we demand the reflection smaller than $0.1 \%$ and the transmission smaller than $3.3 \%$. The similar condition was given in Refs. [14,56,57]. Because the condition is energy dependent, we choose $\Delta r$ and $W_{0}$ as follows:

$$
\Delta r=12 \mathrm{fm}, \quad \eta_{0}=10 \mathrm{MeV} .
$$

This satisfy the condition of Eq. (21) for $7<E<60 \mathrm{MeV}$.

For the linear response calculation, first, we solve the static HF problem with the imaginary-time method [58] to determine the occupied HF orbitals $\left\{\phi_{i}^{0}\right\}_{i=1, \ldots, A}$. Then, an external perturbative field, $V_{\text {ext }}(\mathbf{r}, t)=\epsilon F(\mathbf{r}) \delta(t)$, is turned on instantaneously at $t=0$. This results in an initial state of the TDHF calculation as follows:

$$
\phi_{i}(\mathbf{r}, t=0+)=e^{-i \epsilon F(\mathbf{r})} \phi_{i}^{0}(\mathbf{r}),
$$

where the constant $\epsilon$ is arbitrary but should be small enough to validate the linear approximation of Eq. (3). We calculate time evolution of the expectation value of $F(\mathbf{r})$ (assumed to be real) as follows:

$$
\begin{aligned}
\langle\Psi(t)|F| \Psi(t)\rangle & =\int d \mathbf{r} \sum_{i=1}^{A} \phi_{i}^{*}(\mathbf{r}, t) F(\mathbf{r}) \phi_{i}(\mathbf{r}, t) \\
& =\int d \mathbf{r} F(\mathbf{r}) \delta \rho(\mathbf{r}, t) .
\end{aligned}
$$

Here, we assume that the ground-state expectation value of $F(\mathbf{r})$ is zero at the last equation. Comparing its Fourier transform with Eq. (9), we have the following:

$$
\frac{d B(\omega ; F)}{d \omega}=-\frac{1}{\pi \epsilon} \operatorname{Im} \int d t\langle\Psi(t)|F| \Psi(t)\rangle e^{i \omega t} .
$$

Note that $\langle\Psi(t)|F| \Psi(t)\rangle$ is fully determined by wave functions in the inner space $(r<R)$, as far as the linear approximation is valid. This can be easily understood using a relation, $\delta \rho=$ $\sum_{i} \phi_{i}^{0 *} \delta \phi_{i}+$ h.c., and the condition that $\phi_{i}^{0}=0$ at $r>R$.

\section{B. Adaptive-coordinate 3D grid space}

There is a significant improvement of the computational cost by reducing the number of grid points in the outer space. Although we take the outer model space roughly the same size as the inner one in the radial coordinate $(R \approx \Delta r)$, its volume is considerably larger because the volume element increases as $r^{2} d r$. Therefore, the calculation of wave functions in the outer model space consumes most of the computation time. However, because wave functions in the outer space are irrelevant for $\langle\Psi(t)|F| \Psi(t)\rangle$, the accurate description is not 


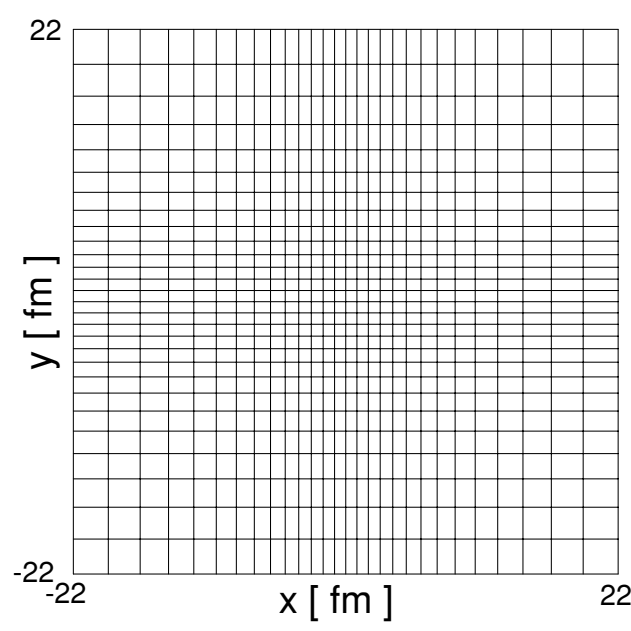

FIG. 1. Adaptive grid in the $(x, y)$ plane for the coordinate transformation of Eq. (26) with $x_{0}=8 \mathrm{fm}, k=5$, and $n=2$. The $(u, v, w)$ space is discretized in square mesh of $0.9 \mathrm{fm}$.

necessary there. Therefore, we use the adaptive curvilinear coordinate in the small-amplitude $\mathrm{TDHF}+\mathrm{ABC}$ calculation [59]. The coordinate transformation we use in this article is as follows:

$$
x(u)=x_{0} \frac{k u / x_{0}}{1+(k-1) u /\left(x_{0} \sinh \left(u / x_{0}\right)\right)^{n}},
$$

and the same form for $y(v)$ and $z(w)$ as well. This function has an asymptotic values, $x(u) \sim u$ at $u \ll x_{0}$ and $x(u) \sim k u$ at $u \gg x_{0}$. All the derivatives and integrals in $(x, y, z)$ space are mapped to those in $(u, v, w)$ space. For instance,

$$
\begin{aligned}
\frac{\partial^{2}}{\partial x^{2}} & =\frac{d^{2} u}{d x^{2}} \frac{\partial}{\partial u}+\left(\frac{d u}{d x}\right)^{2} \frac{\partial^{2}}{\partial u^{2}}, \\
\int d \mathbf{r} & =\int d u d v d w \frac{d x}{d u} \frac{d y}{d v} \frac{d z}{d w} .
\end{aligned}
$$

The 3D $(u, v, w)$ space is discretized in square mesh and finite-point formula in this space is applied to numerical differentiation. The curvilinear grid space employed in Secs. IV and $\mathrm{V}$ is shown in Fig. 1.

\section{ILLUSTRATIVE EXAMPLES: APPLICATION WITH THE BKN INTERACTION}

In this section, some illustrative examples are shown to demonstrate the effects of continuum, the validity of the bound-state $\left(L^{2}\right)$ approximation, and a comparison between Green's function and the ABC approach. We adopt the BKN interaction used in Ref. [47]. Note that, for this schematic interaction, the spin-isospin degeneracy is assumed all the time and the Coulomb potential acts on all orbitals with a charge $e / 2$. The HF one-body Hamiltonian is given by the following:

$$
h[\rho]=-\frac{1}{2 m} \nabla^{2}+\frac{3}{4} t_{0} \rho+\frac{3}{16} t_{3} \rho^{2}+W_{Y}+W_{C},
$$

where the Yukawa potential, $W_{Y}$, and Coulomb potential, $W_{C}$, consist of their direct terms only. The parameters are taken from Table I of Ref. [47].

\section{A. Continuum in the spherical nucleus: ${ }^{16} \mathrm{O}$}

\section{1. $L^{2}$ approximation of the continuum}

RPA calculations are often performed on the $L^{2}$ basis set, such as the harmonic oscillator basis. Bound excited states are well described in those calculations, but how accurate is the $L^{2}$ approximation for resonance and continuum states? In other words, what size of model space is necessary to describe an excited state with a finite life time? In Ref. [17], we give a relation between the box size $R$ and the energy resolution $\Delta E$ for continuum states; $\Delta E \sim \hbar v / R$, where $v$ is the velocity of an escaping particle. If we consider a resonance with a lifetime of $\tau$, we should read $\Delta E \sim \hbar v /(R+v \tau)$. For a long-lived state, $v \tau \gg R$, this is identical to the uncertainty principle, $\Delta E \sim \hbar / \tau$. However, for a state of $v \tau \ll R$, such as broad resonance and nonresonant continuum, the resolution is limited by the size of model space.

Using the BKN interaction, it is easy to perform the self-consistent $1 \mathrm{D}$ continuum RPA calculation for closedshell spherical nuclei. We show, in Fig. 2, results of the 1D continuum RPA and the RPA in a box radius $R$ with BBC, which is referred to as "discretized RPA," for isoscalar (IS) octupole resonance in ${ }^{16} \mathrm{O}$. We should note that a similar study on monopole resonance can be found in Ref. [17]. For the continuum RPA calculation, the outgoing boundary condition is imposed at $r=8 \mathrm{fm}$ (top panels). For simplicity, we use the free asymptotic form, $w_{l}^{( \pm)} \sim e^{ \pm i k r}$ with $k^{2} / 2 m=$ $\left[E-V_{C}(r)-l(l+1) / 2 m r^{2}\right]$ at $r=8 \mathrm{fm}$, instead of the exact Coulomb wave function. For the discretized RPA, the radius of model space is chosen as $R=20 \mathrm{fm}$ (middle panels) and $R=8 \mathrm{fm}$ (bottom panels). Because the discretized RPA produces only discrete peaks, we use a smoothing parameter $\Gamma$, adding an imaginary part, $i \Gamma / 2$, to the real energy $\omega$. In the continuum calculation, though we do not need to smear out the continuum strength, we use the same value of $\Gamma$ to make the resolution as coarse as the discretized RPA.

The continuum RPA calculation clearly shows the lowenergy octupole resonance (LEOR) and high-energy octupole resonance (HEOR). The single-particle energy for $p$ shell is about $-16 \mathrm{MeV}$ in this calculation. Thus, the LEOR is a bound peak whose width is entirely from a smoothing parameter $\Gamma$. As you see in Fig. 2, the bound LEOR peak depends neither on the boundary condition nor on the box size $R$. This justifies the use of the discretized RPA for bound excited states. Conversely, the structure of HEOR, which is embedded in the continuum, strongly depends on values of $R$. Note that the continuum RPA results with $\Gamma=0.5 \mathrm{MeV}$ is almost identical to that with $\Gamma=0$, which means that the width of HEOR is not artificial in contrast to the LEOR. The parameter $\Gamma$ actually controls the energy resolution. For the discretized calculations with $R=20 \mathrm{fm}$, we need $\Gamma \gtrsim 8 \mathrm{MeV}$ to produce roughly identical results to the continuum calculation. For those with $R=$ $8 \mathrm{fm}$, we still see some discrepancy even with $\Gamma=10 \mathrm{MeV}$. To obtain sensible results in the discretized basis, we should average the strength function with $\Gamma$ inversely proportional to the box size. We find an empirical formula, $\Gamma \approx 3(\hbar v / R)$ for this calculation. The continuum results with $\Gamma=0.5 \mathrm{MeV}$ can be reproduced by the discretized calculation if we employ 

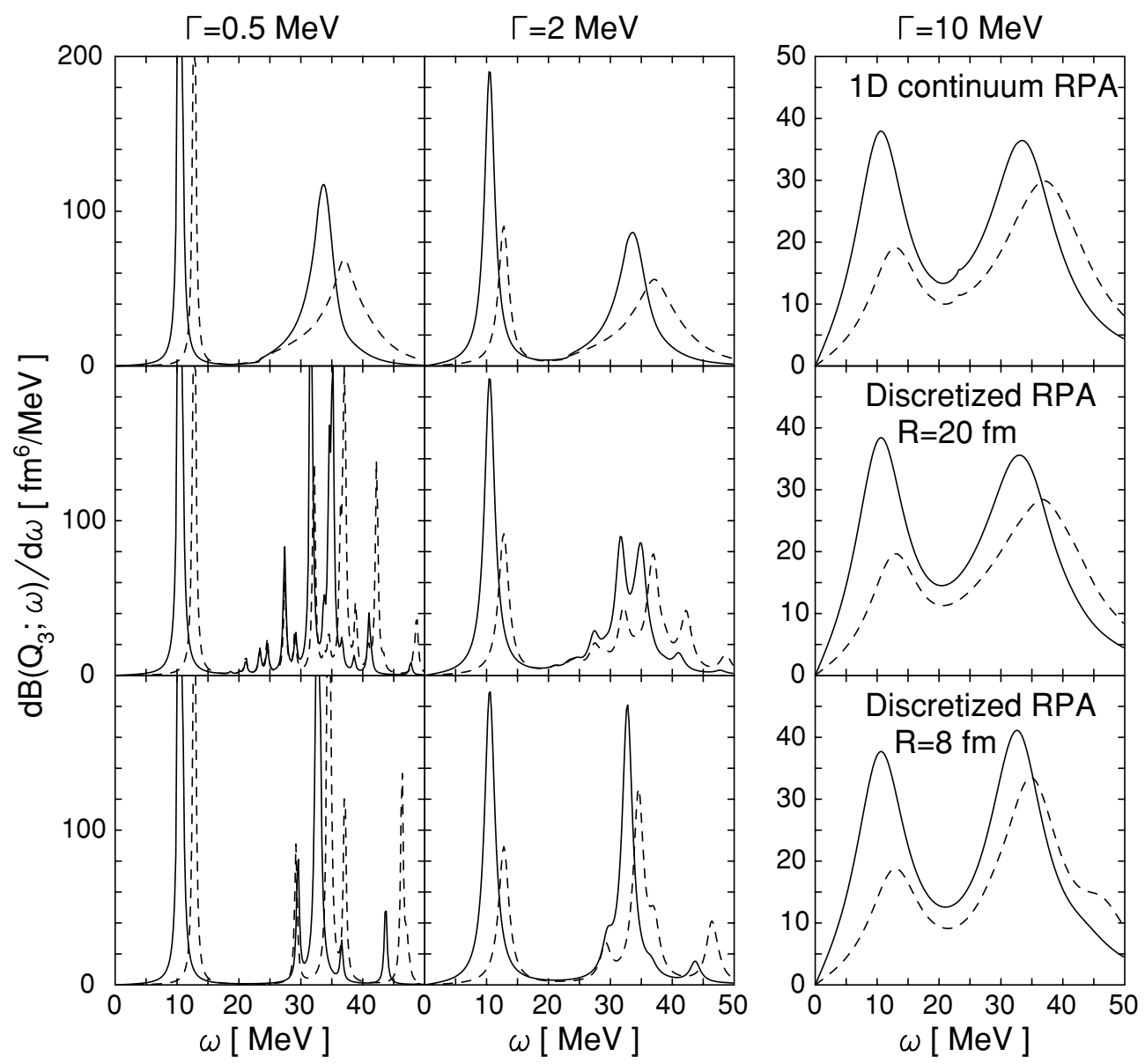

FIG. 2. Mass octupole strengths as functions of excitation energy for ${ }^{16} \mathrm{O}$ calculated with the BKN interaction. The top panels show results of the self-consistent continuum RPA. The middle and bottom panels show results of the discretized RPA of $R=20$ and 8 fm, respectively. The smoothing parameter $\Gamma$ increases from left to right, $0.5,2$, and $10 \mathrm{MeV}$. The solid curves show the RPA strength function, while the dashed show unperturbed one. Because each orbital has a fourfold degeneracy (spin-isospin), the $E 3$ strengths are those shown multiplied by $e^{2} / 4$.

a model space of $R \gtrsim 200 \mathrm{fm}$. It is nearly impossible to treat this size of the $3 \mathrm{D}$ grid space with a present computer power. Therefore, it is certainly desirable to develop a method of treating the continuum boundary condition for deformed nuclei. The methods described in Secs. II and III will serve this purpose. Next, we discuss applications of these methods to the BKN interaction.

\section{Small-amplitude $T D H F+A B C$ vs. continuum RPA}

In this section, we examine accuracy and feasibility of methods in Secs. II and III. We compare results of two methods and show how accurate the TDHF+ABC can be.

We use the same BKN model, Eq. (29), and, again, calculate octupole states in ${ }^{16} \mathrm{O}$. For the $3 \mathrm{D}$ continuum RPA calculation, the model space is the 3D coordinate space of $R=8 \mathrm{fm}$, discretized in square mesh of $\Delta x=\Delta y=\Delta z=1 \mathrm{fm}$. For the real-time method with small-amplitude TDHF+ABC, we use the adaptive curvilinear coordinate of Fig. 1, with $R=8 \mathrm{fm}$ and $\Delta r=12 \mathrm{fm}$ and $\eta_{0}=10 \mathrm{MeV}$ for the absorbing potential. The time evolution is calculated up to $t=30 \mathrm{MeV}^{-1}$, and then we perform the Fourier transform of the time-dependent octupole moment with $F(\mathbf{r})=r^{3} Y_{30}(\hat{\mathbf{r}})$. Results of the Green's function method is shown in Fig. 3(a) and those of the real-time method in Fig. 3(b). These figures are almost identical to each other, but one may notice small difference. First, the strength at $20<\omega<30 \mathrm{MeV}$ is slightly higher for the real-time calculation. This is probably because the condition for the absorber, Eq. (21), breaks down for low-energy particles $(E<$ $7 \mathrm{MeV}$ ). Second, the peak position is higher for the real-time calculation by about $0.3 \mathrm{MeV}$ for LEOR and about $0.6 \mathrm{MeV}$ for HEOR. This is due to the use of adaptive coordinate representation. The time evolution of octupole moment is shown in Fig. 4 with use of the square and the adaptive coordinate. Discrepancy seen at $t>1 \mathrm{MeV}^{-1}$ corresponds to $0.3 \mathrm{MeV}$ difference in the LEOR energy. Comparing results of these calculations with those of the 1D continuum RPA in the radial coordinate, we see a very good agreement (see the top-left panel of Fig. 2). This means that the nucleonic continuum states are properly treated in both calculations of the 3D coordinate space; the Green's function method and the small-amplitude TDHF+ABC. There is a small peak in the $3 \mathrm{D}$ calculation at $\omega=2.5 \mathrm{MeV}$. This is due to small admixture of the spurious translational mode. In Fig. 3(a), the 


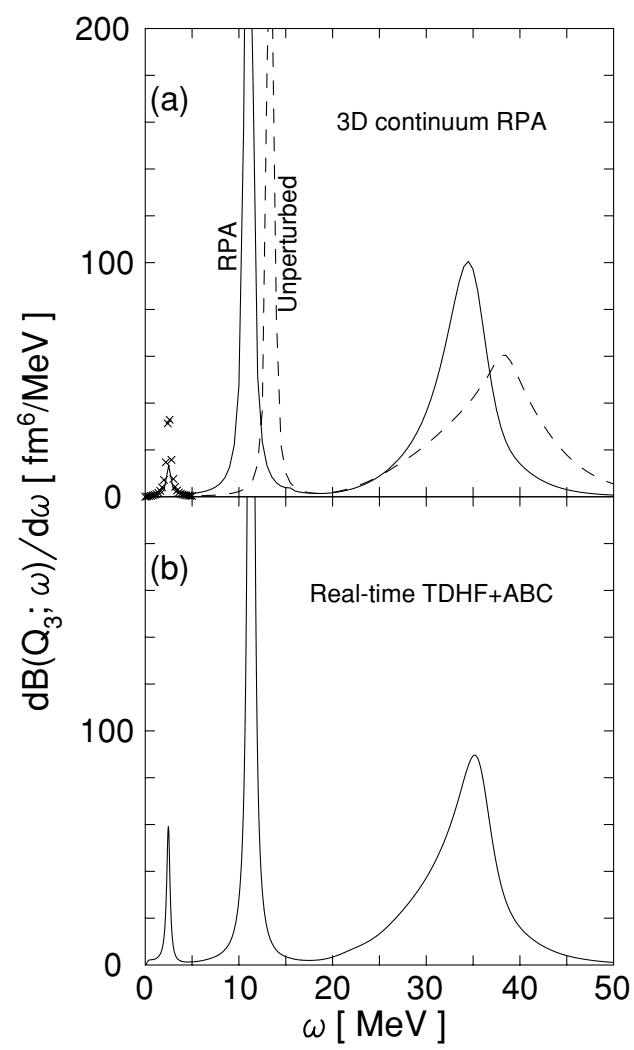

FIG. 3. The same as Fig. 2, but calculated with (a) the Green's function method in the 3D grid space and (b) the real-time smallamplitude $\mathrm{TDHF}+\mathrm{ABC}$ in the adaptive $3 \mathrm{D}$ space. The smoothing parameter is $\Gamma=0.5 \mathrm{MeV}$. Calculated spurious dipole strength is shown by crosses for $0<\omega<5 \mathrm{MeV}$ in units of square femtometers.

strength calculated with $V_{\text {ext }}=r Y_{10}$ is presented by crosses for $0<\omega<5 \mathrm{MeV}$ in units of square femtometers. In the 1D continuum RPA calculation with the partial-wave expansion, these octupole and dipole modes are separated. Thus, this mixing is not present in Fig. 2. However, in the 3D grid space, the translational and rotational invariance of the Hamiltonian is not exact. Adopting finer grid spacing diminishes the spurious peak height and moves its position toward zero energy.

Figure 4 demonstrates an interesting feature in real time. The total energy is conserved within $300 \mathrm{keV}$ up to $t=$ $30 \mathrm{MeV}^{-1}$. With the $\mathrm{BBC}$ instead of the $\mathrm{ABC}$, the energy conservation becomes even better. The absolute scale of its vertical axis does not have a significant meaning because it depends linearly on the arbitrary small parameter $\epsilon$. In the beginning, there is interference between the LEOR and HEOR; however, for $t \gtrsim 1 \mathrm{MeV}^{-1}$, only the LEOR mode survives. This feature clearly indicate stability of the bound collective excitation and decay of the collective mode in the continuum. The single-mode oscillation of the LEOR continues to the end of the time evolution $\left(t=30 \mathrm{MeV}^{-1}\right)$. The HEOR decays into the nucleon emission within time scale of $t \sim 0.5 \hbar / \mathrm{MeV}$. Therefore, a part of the calculated energy width of HEOR, at least a few mega electron volts is associated with this nucleon escape width.

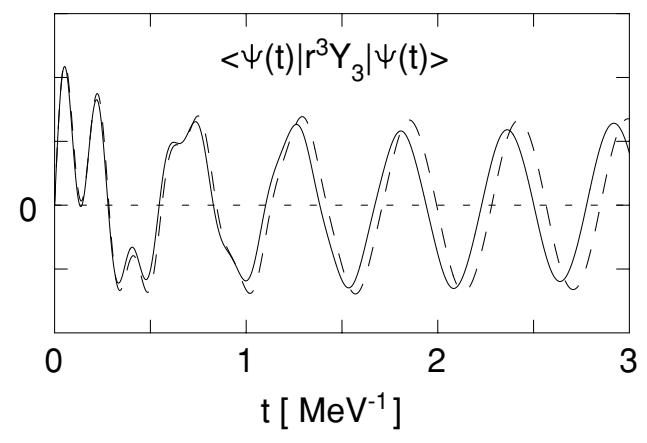

FIG. 4. Time evolution of the octupole moment as a function of time for ${ }^{16} \mathrm{O}$. The calculation in the adaptive (square) mesh coordinate is shown by the solid (dashed) line.

\section{B. Continuum in the deformed nucleus: ${ }^{20} \mathrm{Ne}$}

Now let us discuss a light deformed nucleus, ${ }^{20} \mathrm{Ne}$. This illustrates usefulness and difficulties of the present approaches. Using the $\mathrm{BKN}$ interaction, ${ }^{20} \mathrm{Ne}$ has a superdeformed prolate shape with $\beta \approx 0.6$. This nucleus has a ground-state rotational band and the measured $B\left(E 2 ; 2^{+} \rightarrow 0^{+}\right)$value is consistent with the deformation. Former calculations of the variation after parity projection have produced the $Y_{30}$-type octupoledeformed ground state with the BKN [60] and with the Skyrme interaction [61]. Because the system is deformed, the 1D continuum RPA is no longer applicable. This is the first attempt of the 3D continuum RPA calculation for deformed nuclei.

We use the same model space as the previous calculation on ${ }^{16} \mathrm{O}$. The IS monopole $\left(r^{2}\right)$ and quadrupole field $\left[r^{2} Y_{2 K}(\hat{\mathbf{r}})\right]$ are adopted as the external perturbations in Eq. (4). Results of the 3D continuum RPA are shown in Fig. 5. The calculated singleparticle energy of the last occupied orbital is $-10.8 \mathrm{MeV}$. Thus, all the high-energy peaks in the figure are embedded in the continuum. The giant quadrupole resonance shows three peaks

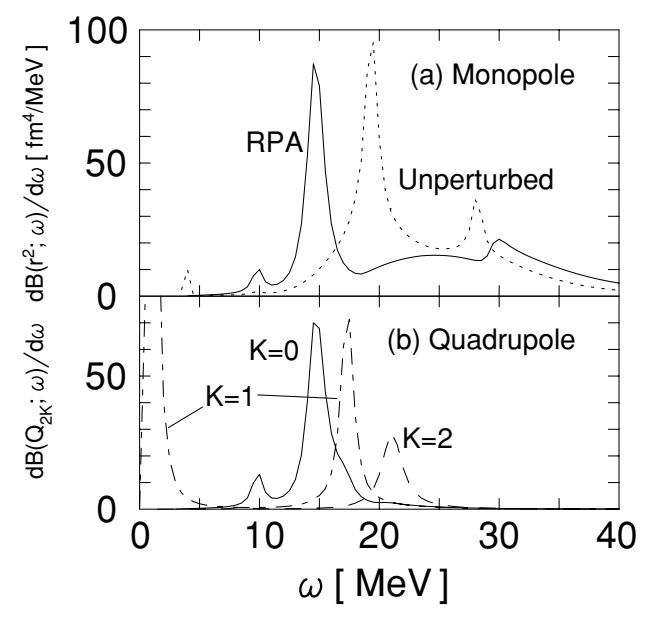

FIG. 5. Calculated strength functions for ${ }^{20} \mathrm{Ne}$. The smoothing parameter $\Gamma=1 \mathrm{MeV}$ is used. (a) IS monopole resonance. The solid (dotted) line indicates the RPA (unperturbed) strengths. (b) IS quadrupole resonance. The $K=0, K=1$, and $K=2$ quadrupole strengths are shown by solid, dash-dotted, and dashed lines, respectively. 
in order of $K=0,1$, and 2 in increasing energy [Fig. 5(b)]. Energy spacing between $K=0$ and 1 peaks is smaller than that between $K=1$ and 2 . This agrees with the simple scaling rule [62]. The result also indicates no low-energy quadrupole vibration except for the zero-mode with $K=1$. This is a characteristic feature in the superdeformation $[63,64]$.

The monopole strength seems to consist of two components: a peak at $15 \mathrm{MeV}$ and a broad hump in the energy region of $E>20 \mathrm{MeV}$. The peak position is lower than that of the unperturbed peak, by about $5 \mathrm{MeV}$. For the monopole strength in ${ }^{16} \mathrm{O}$, calculated strength is shifted higher in energy with the BKN interaction [17]. Therefore, we consider this lowering in energy due to strong coupling to the quadrupole resonance. In fact, the peak lies at exactly the same energy as the $K=0$ quadrupole resonance [Fig. 5(b)]. We have reported a similar result for the oblate nucleus, ${ }^{12} \mathrm{C}$ [65]. Although the BKN interaction may not be realistic for arguing real phenomena in ${ }^{20} \mathrm{Ne}$, effects of such coupling in the continuum between different multipole resonances in deformed nuclei would be an interesting subject in future. There are experimental data on this issue [66-68].

At the end of this section, we mention a numerical problem of the real-time TDHF+ABC method. We have difficulty calculating a certain class of IS modes of excitation with the realtime method. This is associated with zero (Nambu-Goldstone) modes. For instance, calculating TDHF time evolution with the external perturbative IS $K=0 / K=1$ octupole field, the center of mass of the nucleus starts moving because of coupling to the translational motion. Of course, if we adopt a very small grid spacing, these modes are decoupled, which is guaranteed by the self-consistent HF+RPA theory. In practice, we use a mesh of order of $1 \mathrm{fm}$ in the 3D Cartesian coordinates and a finer mesh size drastically increases a computational task. The problem is more serious in deformed cases than in spherical, because the angular momentum selection rule no longer works. In addition, the deformed nucleus has the rotational mode as another zero mode, which is clearly seen in the $K=1$ mode in Fig. 5. In ${ }^{16} \mathrm{O}$, we are able to perform the time evolution up to $t \geqslant 30 \mathrm{MeV}^{-1}$; however, for the $K=0$ octupole mode in ${ }^{20} \mathrm{Ne}, t \approx 10 \mathrm{MeV}^{-1}$ is a limit of time period in which the reliable calculation can be done. This is, of course, a matter of computational cost. If we do not use the adaptive curvilinear coordinate and adopt a larger space, we can carry out a stable calculation for a longer period. Because of this problem, in the next section we discuss applications with the Skyrme interaction to the isovector (IV) giant dipole resonances (GDR), which is more stable and feasible.

\section{GDR STUDIED WITH SKYRME TDHF+ABC}

\section{A. Effects of time-odd mean field in ${ }^{16} \mathrm{O}$}

The continuum RPA with the Skyrme energy functional is a standard method for describing collective excitations in closed-shell spherical nuclei. However, its fully self-consistent calculations have not been achieved in practice, neglecting residual spin-orbit and Coulomb interactions. In addition, some of the time-odd densities, which are known to be important for nuclear moment of inertia and the local Galilean invariance [37], are often neglected in the continuum RPA. In this section, we present an application of the small-amplitude $\mathrm{TDHF}+\mathrm{ABC}$ method to the giant dipole resonance (GDR) in ${ }^{16} \mathrm{O}$. Then, we compare the result with that of the former 1D continuum RPA (which neglected the residual spin-orbit, Coulomb, and spin-spin parts), discussing effects of the residual interactions.

We adopt the Skyrme energy functional as same as Eqs. (A.2), (A.15), and (A.16) in Ref. [70]. The static HF+BCS code based on this functional is called EV8, which assumes the parity and the $z$-signature symmetry. In the present work, we assume no symmetry to allow a time-dependent state to be any Slater determinant during the time evolution. The energy density is written in terms of local densities as follows:

$$
\mathcal{H}(\mathbf{r})=\frac{1}{2 m} \tau(\mathbf{r})+\mathcal{H}^{\text {even }}(\mathbf{r})+\mathcal{H}^{\text {odd }}(\mathbf{r}),
$$

with

$$
\begin{gathered}
\mathcal{H}^{\text {even }}(\mathbf{r})=\mathcal{H}^{\text {even }}\left[\rho^{n}, \rho \triangle \rho, \rho \tau, \rho \stackrel{\leftrightarrow}{\nabla} \cdot \stackrel{\leftrightarrow}{\mathbf{J}}\right], \\
\mathcal{H}^{\text {odd }}(\mathbf{r})=\mathcal{H}^{\text {odd }}\left[\mathbf{j}^{2}, \mathbf{s}^{2}, \mathbf{s} \cdot \nabla \times \mathbf{j}\right] .
\end{gathered}
$$

Here, we follow the notation in Ref. [37]. According to Ref. [70], terms of $\mathbf{s} \cdot \mathbf{T}-\overleftrightarrow{\mathbf{J}}^{2}, \mathbf{s} \cdot \Delta \mathbf{s}$, and $(\nabla \cdot \mathbf{s})^{2}$ are omitted. The energy functional, $\mathcal{H}^{\text {even }}+\mathcal{H}^{\text {odd }}$, keeps the local gauge invariance [36,37]. It is customary in the static HF calculation to take account of the center-of-mass correction by multiplying the first term in Eq. (30) by a factor $(A-1) / A$. We use this correction both for static and dynamic calculations.

To see effects of time-odd components, we adopt the SIII interaction, which was used in the 1D continuum RPA calculation in Ref. [23]. We perform the TDHF calculation with the full functional of $\mathcal{H}^{\text {even }}+\mathcal{H}^{\text {odd }}$ and the one neglecting $\mathcal{H}^{\text {odd }}$. Hereafter, let us call the former functional "SIII-full" and the latter "SIII-even." The instantaneous external field is chosen as follows:

$$
V_{\mathrm{ext}}(\mathbf{r}, t)=\epsilon \mathcal{M}(E 1, \mu=0) \delta(t)=\epsilon e^{(E 1)} r Y_{10}(\hat{\mathbf{r}}) \delta(t),
$$

where $e^{(E 1)}$ indicates the $E 1$ recoil charge, $N e / A$ for protons and $-Z e / A$ for neutrons. $\epsilon$ is an arbitrary small number. Then, solving the TDHF equation as follows:

$$
i \frac{\partial}{\partial t} \phi_{i}(t)=\left\{-\frac{1}{2 m} \nabla^{2}+V^{\text {even }}(t)+V^{\text {odd }}(t)\right\} \phi_{i}(t),
$$

for $i=1, \ldots, A$. The time evolution is performed up to $t=$ $30 \mathrm{MeV}^{-1}$. The time-even mean field, $V^{\text {even }}$, has been well tested against a large number of experimental observations. To test the time-odd mean field, $V^{\text {odd }}$, we need to investigate dynamical properties of nuclei.

Figure 6(a) shows time evolution of calculated $E 1$ dipole moment, $\langle\Psi(t)|\mathcal{M}(E 1)| \Psi(t)\rangle$. In the SIII-even calculation, we see a beating pattern that results in two main peaks of the dashed line in Fig. 6(b). This is in a good agreement with the result of the 1D continuum RPA [23]. However, the inclusion of the time-odd mean field, which is necessary for the Galilean invariance, changes the strength distribution into a single peak (solid line). We decompose effects of time-odd densities into those of current density $\mathbf{j}$ and spin density $\mathbf{s}$ in Figs. 6(c) and (d), 

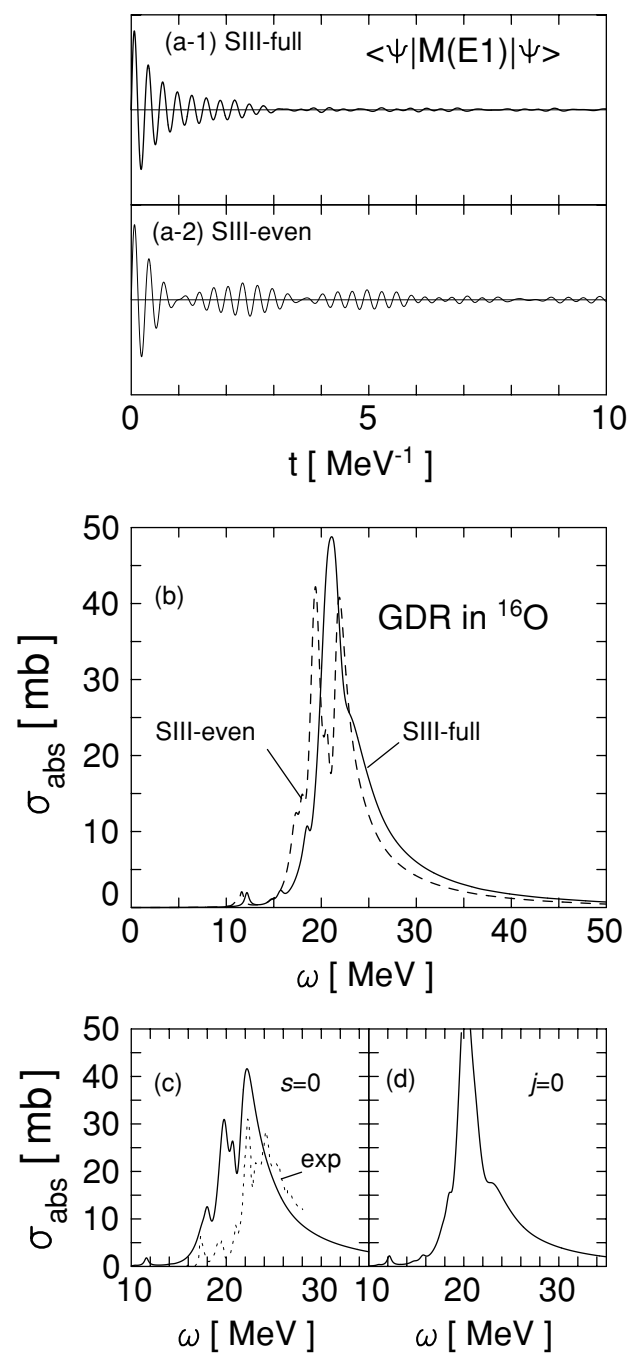

FIG. 6. Results of the Skyrme TDHF+ABC for GDR in ${ }^{16} \mathrm{O}$. (a-1) Time evolution of the $E 1$ moment as a function of time calculated with the SIII-full. (a-2) The same as (a-1) but with SIII-even. (b) Calculated photoabsorption cross section as a function of excitation energy. The SIII-full calculation (solid line) is compared to the SIII-even (dashed line). The smoothing parameter $\Gamma=0.5 \mathrm{MeV}$ is used. (c) The same as (b) but neglecting the spin density s. Experimental photoneutron cross section $(\times 3.5)$ is shown by the dotted line [69]. (d) The same as (b) but neglecting the current density $\mathbf{j}$. See text for details.

respectively. The current density provides additional residual interaction to push the GDR to higher energy by $0.5-$ $1.3 \mathrm{MeV}$, whereas the spin density merges the two main peaks into one. This effect of time-odd density is not special to the SIII interaction. The same effect is observed with the SGII parameters of the Skyrme interaction. See Ref. [71] for a brief report on the same calculation with the SGII force. It is somewhat surprising that not only the current but also the spin density significantly modify the GDR structure. Photoneutron cross-sectional data [69] are shown in Fig. 6(c) with a dotted line. Their absolute values are multiplied by 3.5 , because the data indicate less than $20 \%$ of the TRK sum rule. The experimental shape of the GDR resembles that of the SIII-even calculation, but the two main peaks are calculated lower by about $3 \mathrm{MeV}$. Agreement on the main peak position is slightly improved in the SIII-full calculation, though the calculated peak is still lower than that of the experiment by $2-2.5 \mathrm{MeV}$.

If the interaction commutes with the $E 1$ operator, the oscillator sum is as follows:

$$
\begin{aligned}
S(E 1) & \equiv \int_{0}^{\infty} E B\left(E 1 ; 0^{+} \rightarrow E\left(1^{-}\right)\right) d E, \\
& =\sum_{\mu=-1}^{1} \int_{0}^{\infty} E|\langle E(1 \mu)|\mathcal{M}(E 1, \mu)| 0\rangle|^{2} d E,
\end{aligned}
$$

is identical to the following TRK classical sum rule value:

$$
S(E 1)_{\text {class }}=\frac{9 e^{2}}{8 \pi m} \frac{N Z}{A} .
$$

For ${ }^{16} \mathrm{O}$, the classical sum rule gives $S(E 1)_{\text {class }}=$ $59.4 e^{2} \mathrm{fm}^{2} \mathrm{MeV}$. Because the Skyrme interaction has momentum and isospin dependence, the classical sum rule is violated to a certain extent. We have $S(E 1)=75.1 e^{2} \mathrm{fm}^{2} \mathrm{MeV}$ for SIII-full and $S(E 1)=67.1 e^{2} \mathrm{fm}^{2} \mathrm{MeV}$ for SIII-even. The enhancement of the TRK sum is $26 \%$ for SIII-full and it reduces to $13 \%$ if we neglect the time-odd mean field. This difference mainly comes from the spin density. If we integrate the strength in the energy region up to $30 \mathrm{MeV}$, we have $S(E 1) \approx S(E 1)_{\text {class }}$ for both SIII-full and SIII-even.

\section{B. $E 1$ resonances in even-even $B$ isotopes}

Finally, we apply the small-amplitude TDHF+ABC method to $E 1$ resonances in beryllium isotopes. Beryllium nuclei have been extensively studied both theoretically and experimentally (see, e.g., Ref. [72] and references therein). ${ }^{8} \mathrm{Be}$ is well known for the $\alpha-\alpha$ clustering structure with an elongated prolate shape. Valence neutrons added to ${ }^{8} \mathrm{Be}$ are expected to cause variety of structure change in the ground and excited states [73-75]. ${ }^{10} \mathrm{Be}$ has two neutrons in addition to ${ }^{8} \mathrm{Be}$. The $\alpha-\alpha$ distance in the ground state is considered to be slightly smaller than that in ${ }^{8} \mathrm{Be} .{ }^{12} \mathrm{Be}$ is a semimagic nucleus $(N=8)$; however, its properties are different from spherical closed-shell nuclei. The measured spectroscopic factors suggest that the last neutron pair is two-thirds in the $s d$ configurations [76]. The neighboring odd nucleus, ${ }^{11} \mathrm{Be}$, is famous for the parity inversion and for the halo structure in the ground state. The existence of ${ }^{14} \mathrm{Be}$ at the drip line beyond $N=8$ also indicates weakening of shell closure at $N=8$. Because both $Z=4$ and $N=10$ are the magic numbers at the prolate superdeformed shape, we expect ${ }^{14} \mathrm{Be}$ to be deformed as large as ${ }^{8} \mathrm{Be}$. A new mode of excitation of significant interest is the soft $E 1$ mode near the neutron drip line [77,78]. Coupling in the continuum between the soft $E 1$ mode and the quadrupole deformation is an unsolved problem that can be addressed by the present method to some extent.

We use the Skyrme interaction of the SIII parameters including the time-odd components (SIII-full). The adopted model space is the adaptive grid in Fig. 1 with $R=10 \mathrm{fm}$ and $\Delta r=12 \mathrm{fm}$. Usually, the static HF calculation is carried out with constraint on the center of mass at the origin. However, in this calculation, we impose no condition on the center-of-mass and on the direction of the principal axis. Although this results 
in heavy computation for the imaginary-time step, it turns out that this is important for the stable time evolution of the TDHF state kicked off by the external perturbation. The external field is the same form as Eq. (33) but includes $r Y_{1 \pm 1}$. The TDHF calculation with the perturbative $E 1$ field provides the $E 1$ intrinsic strength, $d B(\omega, \mathcal{M}(E 1, K)) / d \omega$ through Eq. (25). Assuming the strong coupling scheme [46], the $B(E 1)$ transition strength in the laboratory frame is given by the following:

$$
\begin{aligned}
\frac{d B(\omega ; E 1)}{d \omega} & \equiv \int d E_{x} B\left(E 1 ; 0^{+} \rightarrow E_{x}\left(1^{-}\right)\right) \delta\left(\omega-E_{x}\right) \\
& =\sum_{K} \int d E_{x}\left|\left\langle E_{x}|\mathcal{M}(E 1, K)| 0\right\rangle\right|^{2} \delta\left(\omega-E_{x}\right) \\
& =\sum_{K=0, \pm 1} \frac{d B(\omega ; \mathcal{M}(E 1, K))}{d \omega}
\end{aligned}
$$

Here, the state $|0\rangle\left(\left|E_{x}\right\rangle\right)$ is the intrinsic ground (excited) state.

The static HF calculation predicts all these nuclei to be deformed in prolate shape in the ground state. Calculated quadrupole deformations are given in Table I. As we expected, ${ }^{8} \mathrm{Be}$ and ${ }^{14} \mathrm{Be}$ possess large deformation. ${ }^{12} \mathrm{Be}$ has the smallest deformation, but its proton distribution has a moderate deformation. The static $\mathrm{HF}$ analysis on Be isotopes with the SIII force have been already done in Ref. [79]. The total binding energies are well reproduced. Calculated occupied single-particle energies are listed in Table II. In the linear response approximation of the TDHF, the neutron continuum plays its role at energies higher than the absolute value of single-particle energy of the last-occupied neutron. Because the proton orbitals become deeply bound in neutron-rich nuclei, the proton continuum is expected to be less important. However, in these Be isotopes, the protons are important to produce prolate deformation of the mean field.

Now let us discuss dynamical properties of these nuclei. In Fig. 7, calculated time-dependent $E 1$ moment is presented as functions of time. The time evolution is calculated up to $t=$ $30 \mathrm{MeV}^{-1}$. The beginning third of the total period is shown in Fig. 7. Note that the amplitude is magnified by a factor of 10 in the latter half of period. Performing the Fourier transform, calculated $B(E 1)$ transition strength is shown in Fig. 8. In ${ }^{8} \mathrm{Be}$, we observe large splitting of the GDR peak associated with the large quadrupole deformation $(\beta \approx 1)$. The magnitude of splitting is more than $10 \mathrm{MeV}$ and the $K=0$ peak (the oscillation along the symmetry axis) is lowered to around $15 \mathrm{MeV}$ in energy. In Fig. 7(a), the amplitude of the $K=0$ oscillation almost monotonically decays as time

TABLE I. Calculated quadrupole deformation for even-even Be isotopes. The last two columns show deformation of neutron and proton density distribution, $\beta_{n}$ and $\beta_{p}$, separately.

\begin{tabular}{cccc}
\hline \hline & $\beta$ & $\beta_{n}$ & $\beta_{p}$ \\
\hline${ }^{8} \mathrm{Be}$ & 1.07 & 1.06 & 1.09 \\
${ }^{10} \mathrm{Be}$ & 0.39 & 0.33 & 0.49 \\
${ }^{12} \mathrm{Be}$ & 0.12 & 0.07 & 0.22 \\
${ }^{14} \mathrm{Be}$ & 0.74 & 0.77 & 0.66 \\
\hline \hline
\end{tabular}

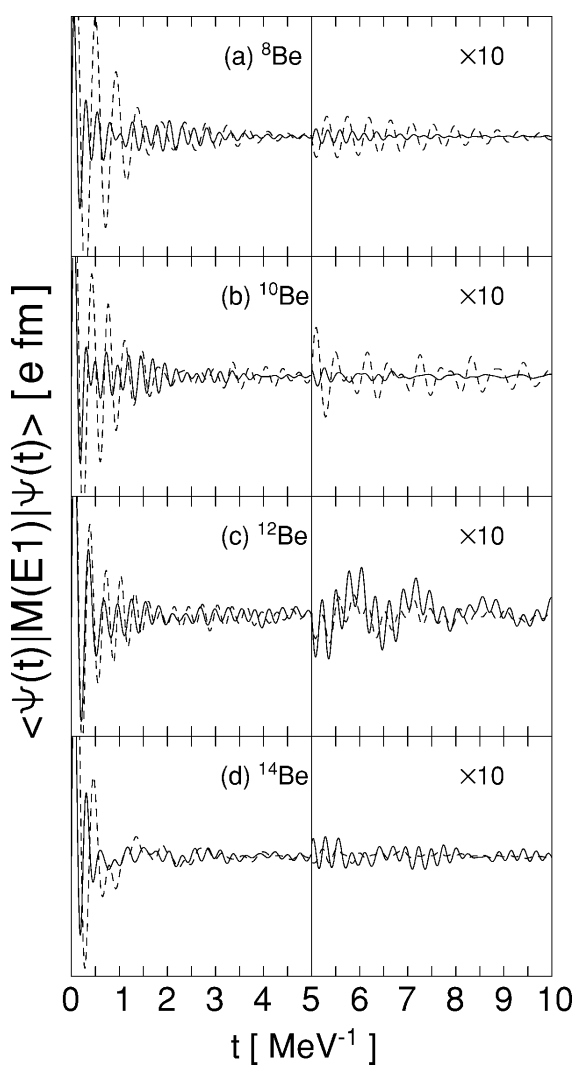

FIG. 7. Calculated $E 1$ moment as functions of time for (a) ${ }^{8} \mathrm{Be}$, (b) ${ }^{10} \mathrm{Be}$, (c) ${ }^{12} \mathrm{Be}$, and (d) ${ }^{14} \mathrm{Be}$. The dashed (solid) line indicates the external field with $K=0(K= \pm 1)$. Scale of the vertical axis is arbitrary because it linearly depends on the small parameter $\epsilon$. It is magnified by a factor of 5 for the latter half of period, $5<t<$ $10 \mathrm{MeV}^{-1}$.

increases, whereas that of the $K=1$ mode shows a beating pattern. This results in a splitting of the high-lying $K=1$ peak. In the total $B(E 1)$ strength function, this is seen as a small peak in the middle of two main peaks. We also see that the $K=0$ oscillation stays longer than the $K=1$. This is because the peak position is near the particle decay threshold, thus, the allowed phase space is smaller for the $K=0$ peak (Table II).

In ${ }^{10} \mathrm{Be}$, we see a similar behavior to that of ${ }^{8} \mathrm{Be}$. Because of the smaller deformation, the lower $K=0$ peak shifts to higher energy by about $5 \mathrm{MeV}$. Figure 8(b) again indicates the $K=1$ mode split into two peaks. Although the ground-state deformation in ${ }^{10} \mathrm{Be}$ is less than half that of ${ }^{8} \mathrm{Be}$, the energy splitting between the lowest and highest peaks is still as large as $7 \sim 8 \mathrm{MeV}$.

Next, let us discuss ${ }^{12} \mathrm{Be}$. The calculated quadrupole deformation is the smallest among these even-even isotopes. In contrast to ${ }^{8,10} \mathrm{Be}$, we see a not distinguished double-peak structure. The GDR shows a peak at $21 \mathrm{MeV}$ with a broader structure around $25 \mathrm{MeV}$. There exists low-energy $E 1$ strength in the continuum below $10 \mathrm{MeV}$. The peak very near zero energy is due to small admixture of the translational mode. Because of this mixing, the response function suffers from spurious oscillatory behavior at energy below $2 \mathrm{MeV}$. Thus, we concentrate our focus on states at $\omega>2 \mathrm{MeV}$. 
TABLE II. Calculated neutron $(n)$ and proton $(p)$ single-particle energies in units of mega electron volts for Be isotopes. Each state has a twofold degeneracy associated with the time-reversal symmetry.

\begin{tabular}{|c|c|c|c|c|c|c|c|}
\hline \multicolumn{2}{|c|}{${ }^{8} \mathrm{Be}$} & \multicolumn{2}{|c|}{${ }^{10} \mathrm{Be}$} & \multicolumn{2}{|c|}{${ }^{12} \mathrm{Be}$} & \multicolumn{2}{|c|}{${ }^{14} \mathrm{Be}$} \\
\hline$n$ & $p$ & $n$ & $p$ & $n$ & $p$ & $n$ & $p$ \\
\hline $\begin{array}{l}-24.8 \\
-13.3\end{array}$ & $\begin{array}{l}-22.9 \\
-11.5\end{array}$ & $\begin{array}{r}-26.3 \\
-12.5 \\
-9.7\end{array}$ & $\begin{array}{l}-31.6 \\
-16.0\end{array}$ & $\begin{array}{r}-25.5 \\
-11.5 \\
-10.3 \\
-4.3\end{array}$ & $\begin{array}{l}-36.7 \\
-20.6\end{array}$ & $\begin{array}{r}-24.9 \\
-14.0 \\
-9.1 \\
-3.5 \\
-2.6\end{array}$ & $\begin{array}{l}-39.1 \\
-25.7\end{array}$ \\
\hline
\end{tabular}

Though the $B(E 1)$ strength in low energy looks small compared to that in the main GDR, the integrated strength in the energy region of $2<\omega<10 \mathrm{MeV}$ amounts to $B(E 1) \approx$ $0.14 e^{2} \mathrm{fm}^{2}$. The lowest sharp peak at $4.5 \mathrm{MeV}$ has $B\left(E 1 ; 0^{+} \rightarrow 1^{-}\right) \approx 0.023 e^{2} \mathrm{fm}^{2}$. The next lowest peak at $5.6 \mathrm{MeV}$ has $B(E 1) \approx 0.027 e^{2} \mathrm{fm}^{2}$. Both peaks have a dominant $K=1$ character. The low-lying $1^{-}$state has been recently observed in ${ }^{12} \mathrm{Be}$ [80]. The observed excitation energy is $E_{x}=$ 2.68(3) MeV with $B\left(E 1 ; 0^{+} \rightarrow 1^{-}\right)=0.051(13) e^{2} \mathrm{fm}^{2}$. Our result is higher in energy by a factor of 2 and the sum of $B(E 1)$ for the lowest two peaks is comparable to the experiment. The two-neutron pairing model in Ref. [81] predicted the $1^{-}$energy very well $(2.7 \mathrm{MeV})$ but overestimated $B(E 1)$ by $\sim 5$ times. The shell model calculation with extended single-particle wave functions in Ref. [82] well reproduced the lowest $1^{-}$state

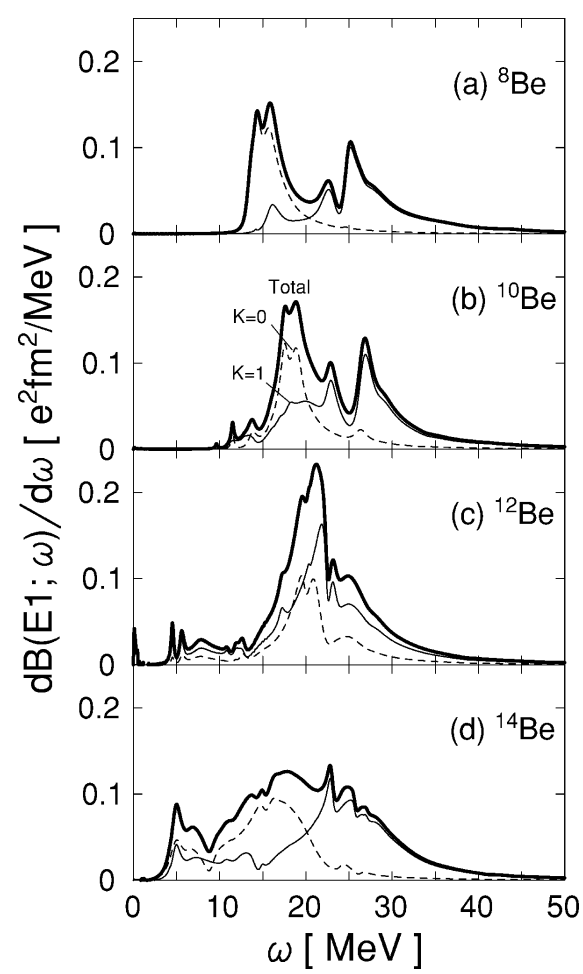

FIG. 8. Calculated values of $d B\left(E 1 ; 0 \rightarrow 1^{-}\right) / d \omega$ for $^{8,10,12,14} \mathrm{Be}$. The smoothing parameter $\Gamma=0.2 \mathrm{MeV}$ is used. The thin dashed (solid) line is a contribution of $K^{\pi}=0^{-}\left(1^{-}\right)$states and the thick solid line for the total strength.
$\left(E_{x}=2.14 \sim 2.9 \mathrm{MeV}\right.$ with $B(E 1)=0.063 \sim 0.072 e^{2} \mathrm{fm}^{2}$ depending on the interaction and model space). They also calculated $B(E 1)$ strength distribution in the GDR energy region without taking account of the continuum. Although their results strongly depend on the adopted interaction and model space, the calculated GDR energy is lower than ours. A striking difference from our result is that they have predicted three main peaks with the WBP interaction. It is not clear at present whether this difference is due to the treatment of the continuum or to the ground-state correlation.

Finally, let us move to the drip line, ${ }^{14} \mathrm{Be}$. The doubly magic closed-shell configuration $(N=10, Z=4)$ at superdeformation leads to the large quadrupole deformation of $\beta=0.74$. The $K=0$ and $K=1$ resonance peaks are at different positions whose centroids are at 15 and $24 \mathrm{MeV}$. Figure 7(d) indicates quick damping of the $K=0$ oscillation. The oscillating pattern almost disappears by $t=$ $3 \mathrm{MeV}^{-1}$. This leads to the large width of the $K=0$ peak in Fig. 8(d). As a consequence of the large width, the double-peak structure in the total $B(E 1)$ strength function is not as clear as in ${ }^{8,10} \mathrm{Be}$. It looks more like a single broad resonance at $20 \mathrm{MeV}$ with the width of about $20 \mathrm{MeV}$. In Fig. 7(d), after the $K=0$ mode disappears, the $K=1$ mode becomes dominant at $t>3 \mathrm{MeV}^{-1}$. This long-lived high-frequency $K=1$ mode results in subpeaks embedded in the broad $K=1$ resonance $(20<\omega<25 \mathrm{MeV})$.

It is known that the weakly bound neutrons strongly couples to the continuum and produces the large dipole strength [83]. The Coulomb breakup of ${ }^{11} \mathrm{Be}$ is a typical example [84]. This is often called "threshold effect," which has a peak at the threshold energy. Because the SIII interaction gives the last neutron binding of $2-3 \mathrm{MeV}$, the threshold effect is weak. In the present calculation, we have not significant threshold strength. Conversely, another soft dipole peak is seen at $5 \mathrm{MeV}$. This peak carries $B(E 1) \approx 0.26 e^{2} \mathrm{fm}^{2}$. A Coulomb dissociation experiment seems to suggest enhanced strength at $E_{x} \approx 2$ and $5 \mathrm{MeV}$ [85]. The shell-model calculation of Ref. [82] also indicates a similar peak $\left[E_{x}=6.76 \sim\right.$ $7.46 \mathrm{MeV}$ with $\left.B(E 1)=0.097 \sim 0.146 e^{2} \mathrm{fm}^{2}\right]$. Using the SGII interaction, this peak is at $7 \mathrm{MeV}$ with $B(E 1) \approx$ $0.14 e^{2} \mathrm{fm}^{2}$ [20], which well agrees with the shell-model result. On the GDR main peaks, our result looks rather different from the shell-model: the shell-model indicates a single main peak at 12-17 MeV, whereas we have a broad resonance whose centroid is around $20 \mathrm{MeV}$. Because the shell model 
TABLE III. Energy-weighted sum rule values in units of $e^{2} \mathrm{fm}^{2}$ $\mathrm{MeV}$. The second column shows values of the classical TRK formula. The small-amplitude $\mathrm{TDHF}+\mathrm{ABC}$ calculation produces values in the third and fourth columns. The fourth column gives the soft $E 1$ strength of the energy-weighted sum, which is defined by $\omega<$ $15 \mathrm{MeV}$. The last column indicates the deformation parameter obtained by the splitting of the GDR peaks.

\begin{tabular}{ccccc}
\hline \hline & $S(E 1)_{\text {class }}$ & $S(E 1)$ & $S(E 1 ; E<15 \mathrm{MeV})$ & $\delta$ \\
\hline${ }^{8} \mathrm{Be}$ & 29.7 & 34.0 & 3.14 & 0.43 \\
${ }^{10} \mathrm{Be}$ & 35.7 & 42.8 & 1.26 & 0.21 \\
${ }^{12} \mathrm{Be}$ & 39.6 & 48.2 & 2.54 & 0.05 \\
${ }^{14} \mathrm{Be}$ & 42.5 & 52.2 & 7.57 & 0.35 \\
\hline \hline
\end{tabular}

also indicates continuous $E 1$ strength in the energy region of $\omega>10 \mathrm{MeV}$, this difference may be simply due to lack of the continuum in Ref. [82].

Calculated TRK sum rule values are listed in Table III. The enhancement is slightly smaller than the spherical ${ }^{16} \mathrm{O}$ case. Among the even-even Be isotopes, the enhancement is the biggest for ${ }^{12} \mathrm{Be}$ whose deformation is the smallest. According to the analysis on ${ }^{16} \mathrm{O}$ in Sec. IV A, about half of this enhancement comes from the effect of the time-odd spin density. The large deformation leads to a strong coupling to the $K$ quantum number, and this may restrict dynamics of spin degrees of freedom. The soft $E 1$ strength, which is defined by the oscillator sum up to $15 \mathrm{MeV}$ in the table, varies among these isotopes. The large value in ${ }^{8} \mathrm{Be}$ is due to the large ground-state deformation that brings the low-energy $K=0$ peak down close to $14 \mathrm{MeV}$. In ${ }^{14} \mathrm{Be}$, it is the largest. This is due to combination of the deformation, the soft dipole peak at $5 \mathrm{MeV}$, and the large width of the $K=0$ resonance at $15 \mathrm{MeV}$. The deformation parameter $\delta$ is estimated from the average energies of $K=0$ and $K=1$ modes. We use Eq. (6-344) in Ref. [46]. The $\delta$ turns out to be much smaller than the deformation of the HF density distribution, $\beta$. The deformation derived from the GDR splitting is known to well agree with that from the E2 moment for actinide nuclei [46]. In light nuclei, the geometrical interpretation of the GDR frequencies may not be justified so well.

\section{CONCLUSION}

We have developed the linear response theory in the continuum applicable to deformed systems. The exact treatment of the continuum is done by the iterative method for construction of the Green's function in the 3D Cartesian grid space (3D continuum RPA). The method is identical to the conventional $1 \mathrm{D}$ continuum RPA in the spherical limit. At the same time, we have shown that the approximate but yet accurate treatment of the continuum can be done by the ABC approach. The small-amplitude $\mathrm{TDHF}+\mathrm{ABC}$ method in the linear response regime is practically identical to the $3 \mathrm{D}$ continuum RPA. Applications of these methods to the TDHF with the BKN interaction reveals their usefulness and accuracy. The real-time TDHF method has a difficulty when we study excitation modes coupled to the zero modes. Because the method is fully self-consistent, the increase of model space (finer grid spacing) will solve the problem, though it requires heavier computation.

Applications to systems with a realistic effective interaction have been performed with the small-amplitude Skyrme $\mathrm{TDHF}+\mathrm{ABC}$. The analysis on the GDR in ${ }^{16} \mathrm{O}$ suggests a significant contribution coming from the time-odd mean field which was often neglected in the 1D continuum RPA. The peak structure in the continuum is affected by these residual interactions, especially by the spin density. Because the spin-dependent terms in the Skyrme energy functional, such as $\mathbf{s}^{2}, \mathbf{s} \cdot \triangle \mathbf{s}$, and $(\nabla \cdot \mathbf{s})^{2}$, are not linked to the time-even components by the local gauge invariance, the analysis may give a useful constraint on these parts of the Skyrme functional.

The coupling to the continuum becomes more important for weakly bound systems. We have studied the deformed continuum of the GDR in Be isotopes. The large deformation splitting of about $10 \mathrm{MeV}$ is predicted for ${ }^{8,14} \mathrm{Be}$. The $K=0$ main peak is significantly lowered by the deformation to less than $15 \mathrm{MeV}$. The time evolution of the $E 1$ moment indicates different damping between ${ }^{8} \mathrm{Be}$ and neutron-rich $\mathrm{Be}$ isotopes, especially for the $K=0$ dipole mode. The soft dipole strength $(E<10 \mathrm{MeV})$ appears in ${ }^{12} \mathrm{Be}$ and ${ }^{14} \mathrm{Be}$. Considering the fact that the SIII parameters were not determined by the isovector properties, we have a reasonable agreement with experiment on the low-energy $1^{-}$state in ${ }^{12} \mathrm{Be}$ and ${ }^{14} \mathrm{Be}$.

In this article, we have studied only the IV GDR in neutronrich nuclei, because of the numerical difficulty discussed above. The IS modes in neutron-rich deformed nuclei are also interesting to investigate. For instance, the octupole correlation in superdeformed ${ }^{14} \mathrm{Be}$ is expected to be stronger than ${ }^{8} \mathrm{Be}$. This is because the superdeformed magic numbers are classified into two category, and the $N=10$ shell closure has a stronger octupole correlation than the $N=4[63,86]$. The small-amplitude TDHF+ABC may be a good method to see how the continuum affects this expectation,

An important extension of the present approaches is the inclusion of pairing. Because the pairing plays an important role in heavy nuclei, this is very desirable but a difficult task. In this respect, we should mention that the HFB-based continuum QRPA has been recently proposed by Matsuo [87] to take account of the continuum for both particle-hole (p-h) and particle-particle (p-p)/hole-hole (h-h) channels. The combination of the 3D continuum RPA and the continuum QRPA may produce a general theory to calculate excited states in the $\mathrm{p}-\mathrm{h}, \mathrm{p}-\mathrm{p}$, and $\mathrm{h}-\mathrm{h}$ continuum for nuclei in the whole nuclear chart.

\section{ACKNOWLEDGMENTS}

This work has been supported by the Grant-in-Aid for Scientific Research in Japan (Nos. 14540369 and 14740146), and has been done as a part of the Japan-U.S. Cooperative Science Program "Mean-field approach to collective excitations in unstable medium-mass and heavy nuclei." A part of the numerical calculations have been performed on the supercomputer at the Research Center for Nuclear Study (RCNP), Osaka University. 
[1] D. Vautherin and D. M. Brink, Phys. Rev. C 5, 626 (1972).

[2] D. Vautherin, Phys. Rev. C 7, 296 (1973).

[3] J. D. Walecka, Ann. Phys. 83, 491 (1974).

[4] J. Dechargé and D. Gogny, Phys. Rev. C 21, 1568 (1980).

[5] D. Lunney, J. M. Pearson, and C. Thibault, Rev. Mod. Phys. 75, 1021 (2003).

[6] J. W. Negele and D. Vautherin, Phys. Rev. C 5, 1472 (1972).

[7] J. W. Negele and D. Vautherin, Phys. Rev. C 11, 1031 (1975).

[8] D. L. Hill and J. A. Wheeler, Phys. Rev. 89, 1102 (1953).

[9] J. J. Griffin and J. A. Wheeler, Phys. Rev. 108, 311 (1957).

[10] D. J. Thouless and J. G. Valatin, Nucl. Phys. 31, 211 (1962).

[11] J. W. Negele, Rev. Mod. Phys. 54, 914 (1982).

[12] E. Runge and E. K. U. Gross, Phys. Rev. Lett. 52, 997 (1984).

[13] E. K. U. Gross, J. F. Dobson, and M. Petersilka, in Density Functional Theory, edited by R. F. Nalewajski (Springer, Heidelberg, 1999), Springer Series Topics in Current Chemistry, pp. 81-172.

[14] T. Nakatsukasa and K. Yabana, J. Chem. Phys. 114, 2550 (2001).

[15] M. Ueda, K. Yabana, and T. Nakatsukasa, Phys. Rev. C 67, 014606 (2002).

[16] M. Ueda, K. Yabana, and T. Nakatsukasa, Nucl. Phys. A738, 288 (2004).

[17] T. Nakatsukasa and K. Yabana, Prog. Theor. Phys. Suppl. 146, 447 (2002).

[18] T. Nakatsukasa, M. Ueda, and K. Yabana, in Proceedings of the International Symposium on Frontiers of Collective Motions (CM2002), edited by H. Sagawa and H. Iwasaki (World Scientific, Singapore, 2003), pp. 267-270.

[19] T. Nakatsukasa and K. Yabana, Eur. Phys. J. A 20, 163 (2004).

[20] T. Nakatsukasa, M. Ueda, and K. Yabana, in Proceedings of the International Conference on the Labyrinth in Nuclear Structure, edited by A. Bracco and C. A. Kalfas (AIP, New York, 2004), pp. 179-183.

[21] S. Shlomo and G. Bertsch, Nucl. Phys. A243, 507 (1975).

[22] A. Zangwill and P. Soven, Phys. Rev. A 21, 1561 (1980).

[23] K. F. Liu and N. van Giai, Phys. Lett. B65, 23 (1976).

[24] J. B. Touv, A. Moalem, and S. Shlomo, Nucl. Phys. A339, 303 (1980).

[25] I. Hamamoto and H. Sagawa, Phys. Rev. C 53, R1492 (1996).

[26] I. Hamamoto, H. Sagawa, and X. Z. Zhang, Phys. Rev. C 55, 2361 (1997).

[27] I. Hamamoto, H. Sagawa, and X. Z. Zhang, Phys. Rev. C 57, R1064 (1998).

[28] I. Hamamoto and H. Sagawa, Phys. Rev. C 60, 064314 (1999).

[29] H. Sagawa, Prog. Theor. Phys. Suppl. 142, 1 (2001).

[30] S. Shlomo and A. I. Sanzhur, Phys. Rev. C 65, 044310 (2002).

[31] B. K. Agrawal, S. Shlomo, and A. I. Sanzhur, Phys. Rev. C 67, 034314 (2003).

[32] B. K. Agrawal and S. Shlomo, Phys. Rev. C 70, 014308 (2004).

[33] T. Nakatsukasa and K. Yabana, Chem. Phys. Lett. 374, 613 (2003).

[34] T. Nakatsukasa and K. Yabana, in Proceedings of the RIKEN Symposium on Physics at Drip Lines, Wako, Japan, 2001, RIKEN Review No. 39 (RIKEN, 2001), pp. 96-101.

[35] G. Bertsch and S. F. Tsai, Phys. Rep. 18, 125 (1975).

[36] Y. M. Engel, D. M. Brink, K. Goeke, S. J. Krieger, and D. Vautherin, Nucl. Phys. A249, 215 (1975).

[37] J. Dobaczewski and J. Dudek, Phys. Rev. C 52, 1827 (1995).

[38] H. Berghammer, D. Vretenar, and P. Ring, Comput. Phys. Commun. 88, 293 (1995).
[39] P. Ring, Prog. Part. Nucl. Phys. 37, 193 (1996).

[40] N. Paar, P. Ring, T. Nikšić, and D. Vretenar, Phys. Rev. C 67, 034312 (2003).

[41] G. Giambrone, S. Scheit, F. Barranco, P. F. Bortignon, G. Colò, D. Sarchi, and E. Vigezzi, Nucl. Phys. A726, 3 (2003).

[42] J. Terasaki, J. Engel, M. Bender, J. Dobaczewski,W. Nazarewicz, and M. Stoitsov (2004), preprint: nucl-th/0407111.

[43] H. Imagawa, Ph.D. thesis, University of Tsukuba (2003).

[44] T. Inakura, M. Yamagami, K. Matsuyanagi, S. Mizutori, H. Imagawa, and Y. Hashimoto, Int. J. Mod. Phys. E 13, 157 (2004).

[45] P. Ring and P. Schuck, The Nuclear Many-Body Problem (Springer-Verlag, New York, 1980).

[46] A. Bohr and B. R. Mottelson, Nuclear Structure, Vol. II (W. A. Benjamin, New York, 1975).

[47] H. Flocard, S. E. Koonin, and M. S. Weiss, Phys. Rev. C 17, 1682 (1978).

[48] P. Bonche, B. Grammaticos, and S. E. Koonin, Phys. Rev. C 17, 1700 (1978).

[49] K. Yabana and G. F. Bertsch, Phys. Rev. B 54, 4484 (1996).

[50] K. Yabana and G. F. Bertsch, Int. J. Quantum Chem. 75, 55 (1999).

[51] G. F. Bertsch, J.-I. Iwata, A. Rubio, and K. Yabana, Phys. Rev. B 62, 7998 (2000)

[52] R. Y. Cusson, J. A. Maruhn, and H. W. Meldner, Phys. Rev. C 18, 2589 (1978).

[53] I. Hamamoto and B. R. Mottelson, J. Phys. Soc. Japan Suppl. 44, 368 (1977).

[54] J. G. Muga, J. P. Palao, B. Navarro, and I. L. Egusquiza, Phys. Rep. 395, 357 (2004).

[55] H. Masui and Y. K. Ho, Phys. Rev. C 65, 054305 (2002).

[56] D. Neuhasuer and M. Baer, J. Chem. Phys. 90, 4351 (1989).

[57] M. Child, Mol. Phys. 72, 89 (1991).

[58] K. T. R. Davies, H. Flocard, S. Krieger, and M. S. Weiss, Nucl. Phys. A342, 111 (1980).

[59] N. A. Modine, G. Zumbach, and E. Kaxiras, Phys. Rev. B 55, 10289 (1997).

[60] S. Takami, K. Yabana, and K. Ikeda, Prog. Theor. Phys. 96, 407 (1996).

[61] H. Ohta, K. Yabana, and T. Nakatsukasa, Phys. Rev. C 70, 014301 (2004).

[62] S. Nishizaki and K. Ando, Prog. Theor. Phys. 73, 889 (1985).

[63] T. Nakatsukasa, S. Mizutori, and K. Matsuyanagi, Prog. Theor. Phys. 87, 607 (1992).

[64] T. Nakatsukasa, K. Matsuyanagi, S. Mizutori, and Y. R. Shimizu, Phys. Rev. C 53, 2213 (1996).

[65] T. Nakatsukasa and K. Yabana, in Proceedings of the 7th International Spring Seminar on Nuclear Physics: Challenges of Nuclear Structure, edited by A. Covello (World Scientific, Singapore, 2002), pp. 91-98.

[66] U. Garg, P. Bogucki, J. D. Bronson, Y.-W. Lui, C. M. Rozsa, and D. H. Youngblood, Phys. Rev. Lett. 45, 1670 (1980).

[67] D. H. Youngblood, Y.-W. Lui, and H. L. Clark, Phys. Rev. C 60, 067302 (1999).

[68] M. Itoh et al., Nucl. Phys. A687, 52c (2001).

[69] B. L. Berman and S. C. Fultz, Rev. Mod. Phys. 47, 713 (1975).

[70] P. Bonche, H. Flocard, and P. H. Heenen, Nucl. Phys. A467, 115 (1987).

[71] T. Nakatsukasa and K. Yabana, in Proceedings of the International Conference on a New Era of Nuclear Structure Physics, 
edited by Y. Suzuki, S. Ohya, M. Matsuo, and T. Ohtsubo (World Scientific, Singapore, 2004), pp. 251-255.

[72] K. Ikeda, I. Tanihata, and H. Horiuchi, eds., Proceedings of the 8th International Conference on Clustering Aspects of Nuclear Structure and Dynamics, Nucl. Phys. A738 (2004).

[73] M. Seya, M. Kohno, and S. Nagata, Prog. Theor. Phys. 65, 204 (1981).

[74] Y. Kanada-En'yo, H. Horiuchi, and A. Ono, Phys. Rev. C 52, 628 (1995).

[75] N. Itagaki, S. Okabe, and K. Ikeda, Phys. Rev. C 62, 034301 (2000).

[76] A. Navin et al., Phys. Rev. Lett. 85, 266 (2000).

[77] P. G. Hansen and B. Jonson, Europhys. Lett. 4, 409 (1987).
[78] K. Ikeda, Nucl. Phys. A538, 355c (1992).

[79] S. Takami, K. Yabana, and K. Ikeda, Prog. Theor. Phys. 94, 1011 (1995).

[80] H. Iwasaki et al., Phys. Lett. B491, 8 (2000).

[81] A. Bonaccorso and N. V. Mau, Nucl. Phys. A615, 245 (1997).

[82] H. Sagawa, T. Suzuki, H. Iwasaki, and M. Ishihara, Phys. Rev. C 63, 034310 (2001).

[83] J. M. Blatt and V. F. Weisskopf, Theoretical Nuclear Physics (Wiley, New York, 1952).

[84] T. Nakamura et al., Phys. Lett. B331, 296 (1994).

[85] M. Labiche et al., Phys. Rev. Lett. 86, 600 (2001).

[86] W. Nazarewicz and J. Dobaczewski, Phys. Rev. Lett. 68, 154 (1992).

[87] M. Masuo, Nucl. Phys. A696, 371 (2001). 